\title{
Magnetic Susceptibility from Quantitative Susceptibility Mapping Can Differentiate New Enhancing from Nonenhancing Multiple Sclerosis Lesions without Gadolinium Injection
}

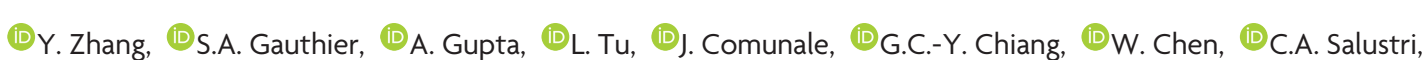

(1)W. Zhu, and (1)Y. Wang to-

\begin{abstract}
BACKGROUND AND PURPOSE: Magnetic susceptibility values of multiple sclerosis lesions increase as they change from gadoliniumenhancing to nonenhancing. Can susceptibility values measured on quantitative susceptibility mapping without gadolinium injection be used to identify the status of lesion enhancement in surveillance MR imaging used to monitor patients with MS?
\end{abstract}

MATERIALS AND METHODS: In patients who had prior MR imaging and quantitative susceptibility mapping in a current MR imaging, new T2-weighted lesions were evaluated for enhancement on conventional T1-weighted imaging with gadolinium, and their susceptibility values were measured on quantitative susceptibility mapping. Receiver operating characteristic analysis was used to assess the diagnostic accuracy of using quantitative susceptibility mapping in distinguishing new gadolinium-enhancing from new nonenhancing lesions. A generalized estimating equation was used to assess differences in susceptibility values among lesion types.

RESULTS: In 54 patients, we identified 86 of 133 new lesions that were gadolinium-enhancing and had relative susceptibility values significantly lower than those of nonenhancing lesions $(\beta=-17.2 ; 95 \% \mathrm{Cl},-20.2$ to $-14.2 ; P<.0001)$. Using susceptibility values to discriminate enhancing from nonenhancing lesions, we performed receiver operating characteristic analysis and found that the area under the curve was $0.95(95 \% \mathrm{Cl}, 0.92-0.99)$. Sensitivity was measured at $88.4 \%$, and specificity, at $91.5 \%$, with a cutoff value of 11.2 parts per billion for quantitative susceptibility mapping-measured susceptibility.

CONCLUSIONS: During routine MR imaging monitoring to detect new MS lesion activity, quantitative susceptibility mapping can be used without gadolinium injection for accurate identification of the BBB leakage status in new T2WI lesions.

ABBREVIATIONS: $\mathrm{Gd}$ = gadolinium; GRE = gradient echo; $\mathrm{ppb}$ = parts per billion; $\mathrm{QSM}$ = quantitative susceptibility mapping

M ultiple sclerosis is an inflammatory disease of the central nervous system, characterized by focal T-cell and macrophage infiltrates associated with demyelination. ${ }^{1,2}$ Because stages of relapse and remission alternate during disease progression, ${ }^{3}$

Received December 27, 2015; accepted after revision April 15, 2016.

From the Department of Radiology (Y.Z., W.C., W.Z.), Tongji Hospital, Tongji Medical College, Huazhong University of Science and Technology, Wuhan, China; Department of Radiology (Y.Z., C.A.S., Y.W.), Weill Cornell Medical College, New York, New York; Departments of Neurology (S.A.G.) and Radiology (A.G., J.C., G.C.-Y.C.), Weill Cornell Medical College, New York-Presbyterian Hospital, New York, New York; School of Applied and Engineering Physics (L.T.) and Department of Biomedical Engineering (Y.W.), Cornell University, Ithaca, New York; and Institute of Cognitive Sciences and Technologies (C.A.S.), Fatebenefratelli Hospital, Rome, Italy.

Authors contributed to this work in the following manner: Ms Yan Zhang, acquisition of data, analysis and interpretation of data, drafting the manuscript; Dr Susan Gauthier, conception and design, acquisition of data, clinical study, revision of the manuscript; Drs Ajay Gupta, Weiwei Chen, Joseph Comunale, and Gloria Chia-Yi Chiang, acquisition of data, interpretation of image data, revision of the manuscript; Mr Lijie Tu, acquisition of data, analysis of data, revision of the manuscript; Dr Carlo Salustri, conception and design, analysis and interpretation of data, revision of the manuscript; Dr Wenzhen Zhu, conception and design, revision of the manuscript, study supervision; and Dr Yi Wang, study concept and design, critical revision of the manuscript for important intellectual content, study supervision. identification and characterization of active lesions are critical for correct diagnosis and therapy. ${ }^{4}$ In clinical practice, current active lesion assessment is based on gadolinium (Gd) enhancement on T1-weighted (T1WI+Gd) MR imaging. However, because Gd enhancement reflects leakage of the blood-brain barrier, it is only an indirect measure of inflammation that is preceded and outlasted by infiltration of immune cells. ${ }^{5}$ The activation of resident innate immune cells may not be captured on T1WI+Gd. ${ }^{6}$ In ad-

All authors had the final approval of the version to be published and agreement to be accountable for all aspects of the work in ensuring that questions related to the accuracy or integrity of any part of the work are appropriately investigated and resolved.

This work was supported by the US Department of Health and Human Services, National Institutes of Health-National Institute of Neurological Disorders and Stroke, grant number R01 NS090464; US Department of Health and Human Services, National Institutes of Health-National Institute of Biomedical Imaging and Bioengineering, grant number R01 EB013443; and the National Natural Science Foundation of China, grant number 81401390.

Please address correspondence to Yi Wang, PhD, Department of Radiology, Weil Cornell Medical College, 515 East 71th St, S-104, New York, NY 10021; e-mail: yiwang@med.cornell.edu

- Indicates open access to non-subscribers at www.ajnr.org

http://dx.doi.org/10.3174/ajnr.A4856 
dition, concerns over repeat Gd exposure have recently been raised in light of new data showing long-term Gd retention in the brains of patients who have undergone multiple Gd injections, ${ }^{7,8}$ including patients with MS in whom Gd retention seems to be associated with degradation into secondary progression. ${ }^{9}$ There has been interest in identifying Gd-enhancing MS lesions without the use of a contrast agent to reduce scan time, cost, and Gd contraindications. ${ }^{10-13}$

It is known that microglia and macrophages in an alternative activation (M2 types) remove myelin debris from MS lesions where they enter peripheral circulation ${ }^{14-17}$; the classic proinflammatory activation (M1 type) tends to accumulate iron. ${ }^{18}$ Both myelin debris removal from and iron accumulation in active MS lesions increase lesion magnetic susceptibility. Analyses of tissue susceptibility changes in sensitive tissues by using gradientecho (GRE) MR imaging have demonstrated that during lesion development, the magnetic susceptibility of an MS lesion as measured on quantitative susceptibility mapping (QSM) increases rapidly as the lesion changes from gadolinium-enhancing to nonenhancing. ${ }^{19-21}$ This finding suggests that during MS lesion development, changes in the Gd-enhancing pattern on T1WI can be indicated by a susceptibility change measured on QSM. Accordingly, this study was designed to assess whether QSM is a viable technique to identify new enhancing MS lesions without Gd injection.

\section{MATERIALS AND METHODS}

The Weill Cornell Medical College institutional review board approved this retrospective study and waived the requirement for informed consent.

\section{Patient Population}

We examined MR images of patients with MS from August 2011 to January 2015 with at least 2 successive MR imaging sessions that included T2-weighted, Gd-enhanced T1-weighted, and GRE imaging. QSM was constructed in an automated manner from GRE data by deconvolving phase with the dipole kernel that connects tissue susceptibility with the magnetic field estimated from the MR imaging phase. ${ }^{19,22,23}$ We compared the lesions on 2 successive MRIs and identified patients with at least 1 new T2WI lesion (ie, a lesion that was not present in prior brain MR imaging in a follow-up MR imaging that was $<1$ year from the baseline MR imaging). All the new lesions were then grouped into enhancing and nonenhancing on T1WI+Gd images.

\section{MR Imaging Examination Protocol}

All examinations were performed on a 3T MR imaging scanner (Signa HDxt; GE Healthcare, Milwaukee, Wisconsin) with an 8-channel head coil. The sequences for each patient were the following: T2WI fast spin-echo, pre- and postgadolinium 3D inversion recovery-prepared T1WI fast spoiled gradient-echo, and 3D $\mathrm{T} 2{ }^{\star} \mathrm{WI}$ spoiled multiecho GRE. Imaging parameters for the multiecho GRE sequence were as follows: TR, $57 \mathrm{~ms}$; number of echoes, 11; first TE, $4.3 \mathrm{~ms}$; TE spacing, $4.8 \mathrm{~ms}$; flip angle, $20^{\circ}$; bandwidth, $244 \mathrm{kHz}$; FOV, $24 \mathrm{~cm}$; matrix, $416 \times 320$; section thickness, $2 \mathrm{~mm}$. The GRE sequence was performed before Gd injection. The total imaging time was 16 minutes 30 seconds.
QSM was constructed from GRE data by using the morphology-enabled dipole inversion. ${ }^{24}$ The images obtained by the other modalities were registered to QSM by using the FMRIB Linear Image Registration Tool (FLIRT; http://www.fmrib.ox.ac.uk). ${ }^{25}$

\section{Data Analysis}

After localizing all new T2WI lesions by comparing them with their previous MRIs, 3 neuroradiologists (J.C., A.G., and G.C.Y.C, with 18,9 , and 8 years of experience, respectively) used the T1WI+Gd images to classify those lesions as enhancing or nonenhancing. They also classified all lesions on QSM as hyperintense and isointense relative to the adjacent white matter. All differences in lesion classification were resolved by the majority.

One neuroradiologist (Y.Z., with 4 years of experience) drew the areas of each localized lesion on the T2WI while blinded to the Gd-enhancement classification. White matter regions without abnormal signal on T1WI and T2WI were identified as normalappearing white matter. For a zero reference, an ROI was chosen on the normal-appearing white matter at the contralateral mirror site of an identified lesion with a similar shape and size on T2WI. Then, the ROIs of lesions and normal-appearing white matter references were overlaid on the QSM images by using a semiautomatic software to assess the values of lesion susceptibility. Veins or artifacts inside the ROIs were excluded by inspection.

\section{Statistical Analysis}

Using relative susceptibilities as a means for distinguishing enhancing from nonenhancing lesions, we assessed the receiver operating characteristic to determine sensitivity, specificity, and the optimal cutoff susceptibility value (in parts per billion [ppb]). Bootstrapped estimates of the area under the curve and 95\% confidence intervals were produced to evaluate variance. The jackknife cross-validation technique was used to evaluate predictive performance of the model. A generalized estimating equation was used to predict QSM values from 3 lesion types: nodular, shell, and nonenhancing. This model assumes a Gaussian distribution and an exchangeable correlation structure to account for the multiple lesions per patient. The generalized estimating equation analysis was also used to predict QSM values from enhancing and nonenhancing lesions, accounting for repeat measurements per patient. All statistical analyses were performed by using SPSS for Windows (Version 16.0; IBM, Armonk, New York). $P<.05$ was considered statistically significant. The accuracy for identifying patients with enhancing lesions was also calculated.

\section{RESULTS}

From the eligible 482 patients with MS, we identified 55 patients with at least 1 new T2WI lesion; there were 133 new T2WI lesions. (One patient was excluded because of motion artifacts on GRE images.) The mean age of the 54 remaining patients ( 11 men and 43 women) was 34.7 years \pm 8.1 (range, $20-52$ years). The disease duration for these patients ranged from 0 to 18 years (mean, $5.71 \pm 4.51$ years) and the Expanded Disability Status Scale scores ranged from 0 to 6 . The Table shows the demographics of these patients.

On T1WI+Gd, 86 (64.7\%) of the 133 lesions from 33 patients were identified as enhancing, and 47 (35.3\%), as nonenhancing 
from 25 patients ( 4 patients had both enhancing and nonenhancing lesions), with complete agreement among the 3 readers. For enhancing lesions, $69(80.2 \%)$ of 86 were found to be isointense on QSM, and 17 (19.8\%), slightly hyperintense in contrast to adjacent white matter. According to their enhancement on T1WI+Gd, the enhancing lesions were divided into 69 nodular and 17 shell. Thirteen of the 17 hyperintense enhancing lesions were shell-enhancing. All 47 nonenhancing lesions were hyperintense on QSM, but 4 (8.5\%) of them were only slightly hyperintense. Sample images are illustrated in Fig 1.

The mean susceptibility of the lesions relative to normalappearing white matter was $20.26 \pm 7.55 \mathrm{ppb}$ for nonenhancing lesions and $2.49 \pm 6.39 \mathrm{ppb}$ for enhancing lesions (both nodular and shell), and their distributions are illustrated by histograms in

\section{Patient demographics}

\begin{tabular}{lccc}
\hline & $\begin{array}{c}\text { Patients with } \\
\text { Enhancing } \\
\text { Lesions }\end{array}$ & $\begin{array}{c}\text { Patients with } \\
\text { Nonenhancing } \\
\text { Lesions }\end{array}$ & P Value \\
\hline No. of patients & 33 & 25 & \\
Sex (F/M) & $28: 5$ & $18: 7$ & \\
Age (yr) (mean) & $36.24 \pm 8.37$ & $32.40 \pm 6.43$ & .07 \\
Disease duration & $5.85 \pm 4.49$ & $5.32 \pm 4.05$ & .65 \\
$\quad$ (yr) (mean) & & & .66 \\
EDSS (mean) & $1.70 \pm 1.57$ & $1.50 \pm 1.69$ & \\
\hline
\end{tabular}

Note:-EDSS indicates Expanded Disability Status Scale.

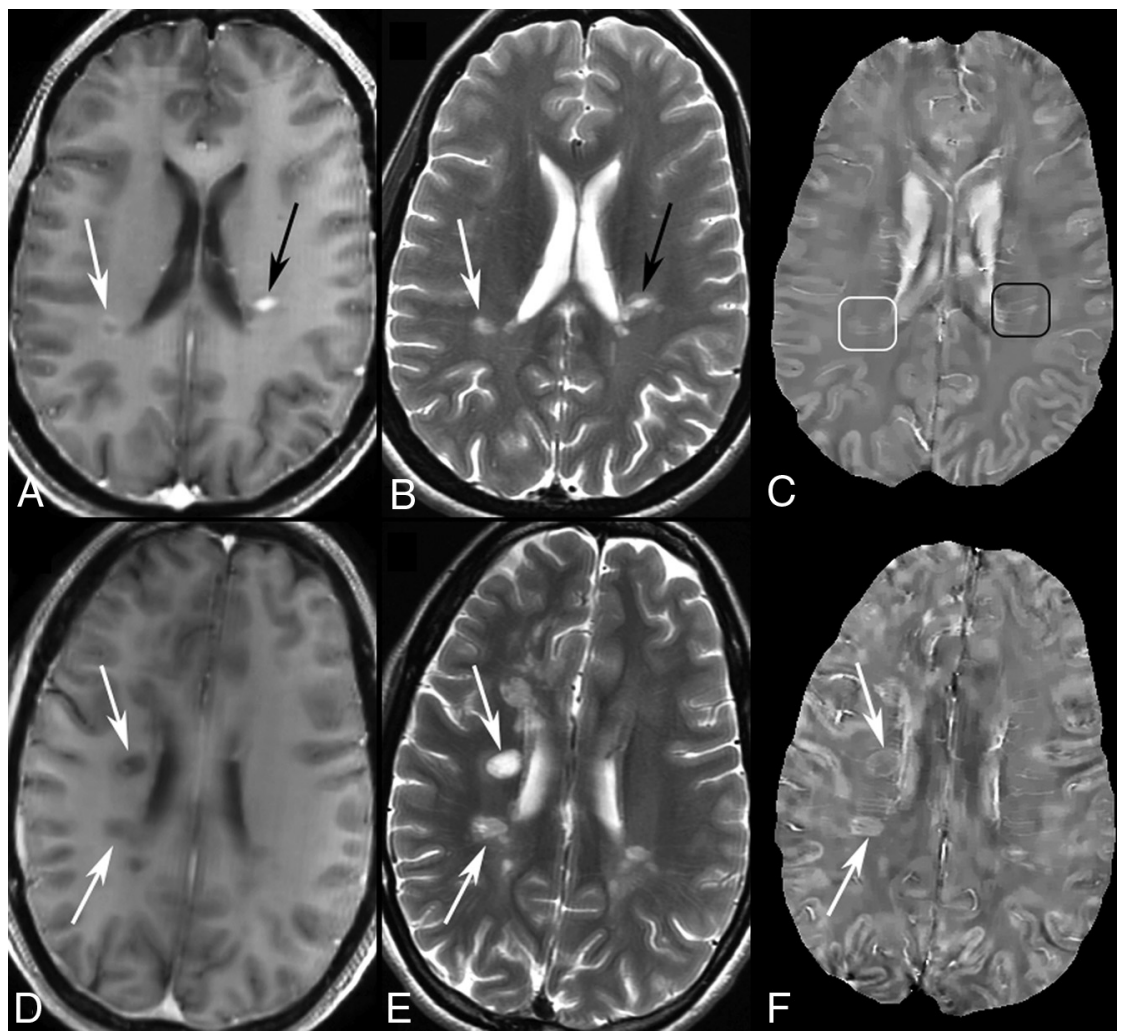

FIG 1. MR images of enhancing and nonenhancing new MS lesions. TIWI+Gd $(A), T 2 W I(B)$, and QSM $(C)$ in a 44-year-old woman with relapsing-remitting MS. Two enhancing lesions $(A$ and $B$, arrows) are found in TIWI+Gd. One is shell-enhancing ( $A$, white arrow) and another is nodularenhancing ( $A$, black arrow). The shell-enhancing lesion appears slightly QSM-hyperintense (C, white box) and the nodular one appears QSM isointense (C, black box). TIWI+ Gd (D), T2WI (E), and QSM $(F)$ in a 35-year-old woman with relapsing-remitting MS. Two new nonenhancing lesions ( $D$ and $E$, arrows) are found in T1WI+Gd and T2WI compared with MR imaging 6 months prior. The 2 lesions both appear QSM-hyperintense with bright rims ( $F$, arrows).
Fig 2. In the generalized estimating equation analysis of lesion susceptibility values among the 3 lesion types, both nodularenhancing $(\beta=-19.6$; $95 \% \mathrm{CI},-23.5$ to $-15.8 ; P<.0001)$ and shell-enhancing lesions $(\beta=-13.5 ; 95 \% \mathrm{CI},-19.0$ to -8.0 ; $P<.0001$ ) had significantly lower susceptibility values compared with nonenhancing lesions. In the generalized estimating equation analysis of susceptibility values between enhancing and nonenhancing lesions, enhancing lesions had significantly lower susceptibility values compared nonenhancing lesions $(\beta=-17.2$; $95 \% \mathrm{CI},-20.2$ to $-11.2 ; P<.0001)$. The exchangeable correlation coefficient was 0.12 for the lesion-susceptibility model.

The receiver operating characteristic curve constructed from the mean relative susceptibility values of lesions is shown in Fig 3. The cross-validated area under the curve was 0.9530 (95\% CI, 0.9201-0.9859) and the bootstrapped area under the curve was 0.9594 (95\% CI, 0.9305-0.9884) for identifying enhancing lesions from QSM-measured susceptibility values. A relative susceptibility cutoff of $11.2 \mathrm{ppb}$ to distinguish enhancing from nonenhancing lesions had a sensitivity and specificity of $88.4 \%$ and $91.5 \%$, respectively.

\section{DISCUSSION}

Our data suggest that QSM and T2WI together allow accurate identification of enhancing lesions in patients with MS without Gd injection within new lesions on serial MR imaging. This may be a potential clinical application of the reported observation that the magnetic susceptibility of an MS lesion increases rapidly as it changes from $\mathrm{Gd}$-enhancing to nonenhancing. ${ }^{19,21}$ Our study suggests that in serial MR imaging during regular monitoring of patients with MS, QSM may a substitute for Gd enhancement in assessing inflammatory activity.

Enhancement on T1WI+Gd is the current standard method to assess ongoing CNS inflammation for monitoring optimizing inflammation-suppressing treatment. Following the initial inflammatory reaction, the $\mathrm{BBB}$ opens and immune cells infiltrate the brain for about 3 weeks; therefore, T1WI+Gd may only offer a small window into lesion pathology. ${ }^{26}$ During this period, the microglia and macrophages take up and degrade myelin fragments; this process is reflected in the initial lack of change in the susceptibilities of active lesions on QSM. However, after the BBB seals, immune cells remain active in the brain tissue. ${ }^{17}$ For example, microglia and macrophages remove diamagnetic myelin fragments, and at the same time or afterward, microglia and macrophage cells with paramagnetic iron gather both at the periphery and within a lesion to further promote inflammation. ${ }^{16}$ Thus, both myelin debris removal and iron ac- 


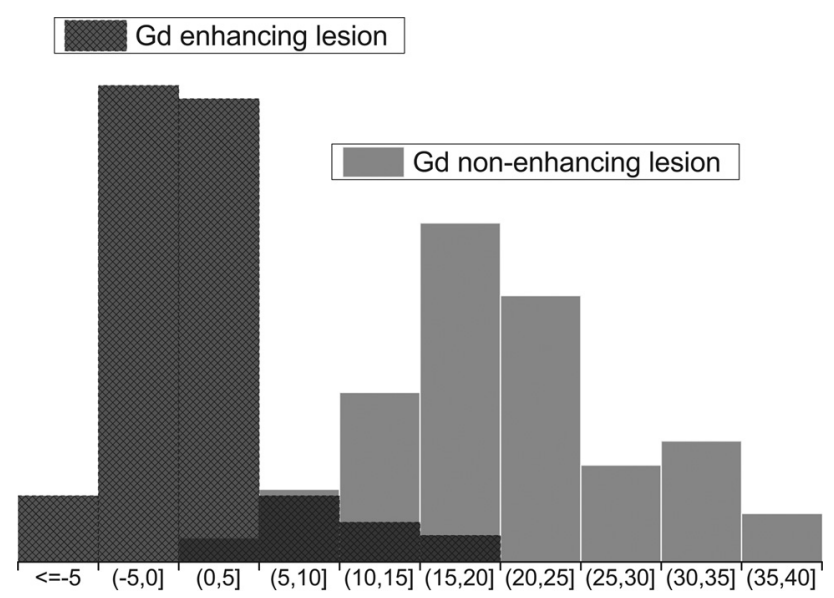

FIG 2. Susceptibility value histogram of enhancing and nonenhancing new lesions. The $\mathrm{x}$-axis is the susceptibility value in parts per billion.

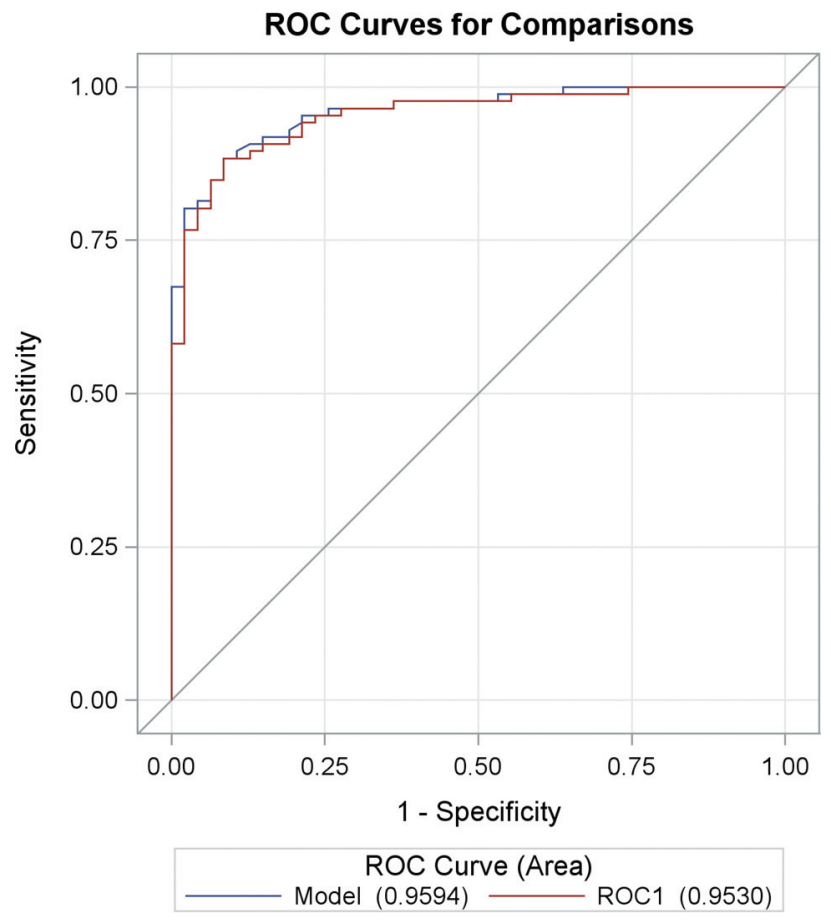

FIG 3. Receiver operator characteristic curves for susceptibility relative to normal-appearing white matter to predict lesion-enhancing status. The area under the curve is 0.9594 from bootstrapped model and 0.9530 from the jackknife cross-validated ROCl.

cumulation likely contribute to the increase in lesion susceptibility observed on QSM. MS lesions are hyperintense for a few years, typically with bright rims on $\mathrm{QSM}^{19}$; these bright rims can be interpreted as iron. ${ }^{27}$ Therefore, including QSM rather than Gd enhancement alone, in an MR imaging protocol for patients with MS may provide more detailed insight into early lesion dynamics in MS.

There has been interest in reducing scan time and cost when identifying the BBB leakage without Gd injection. ${ }^{10-13}$ Getting rid of the Gd injection may be necessary for patients with known contraindications to $\mathrm{Gd}$, including those patients who are allergic to Gd or pregnant. Furthermore, the long-term safety of repeat Gd injections has undergone scrutiny by the FDA because of recent reports showing Gd accumulation in the brains of patients with normal kidney function ${ }^{7,8}$ (http://www.fda.gov/Safety/ MedWatch/SafetyInformation/SafetyAlertsforHumanMedical Products/ucm456012.htm). The mechanism of Gd retention is not yet fully understood but may involve the Gd ion disassociating with the chelator in the contrast agent and binding to metal transporter and storage proteins in brain tissue. Of particular concern is that Gd accumulation in MS brains seems to be associated with degradation into secondary progression. ${ }^{9}$ Therefore, alternative imaging strategies that accurately characterize MS disease activity without Gd should be actively investigated, established, and disseminated to the MS community. Previous effort in identifying Gd-enhancing lesions has not been satisfactory, yielding a diagnostic accuracy of an area under the curve of 0.83 in receiver operating characteristic analysis by using semiquantitative and quantitative T1WI and T2WI ${ }^{10,12}$ and an accuracy of $72.1 \%$ by using diffusion-weighted imaging. ${ }^{13}$ Fundamentally, relaxation time and the diffusion coefficient are proportional to the correlation time, which reflects cellular content in a voxel and cannot differentiate Gd-enhancing and nonenhancing lesions. QSM used in this work reflects myelin debris removal and iron accumulation in MS lesions and improves the diagnostic accuracy to an area under the curve of 0.96 , which may be accurate enough to serve as an alternative method for monitoring new inflammatory activity in patients with MS without Gd injection.

QSM used in this study is processed from complex data (both real and imaginary or both magnitude and phase) acquired in gradient-echo MR imaging. ${ }^{23}$ Because of its sensitivity to magnetic susceptibility, GRE has been used in previous studies to observe MS lesions. ${ }^{19,20,28-36}$ There are many ways to process or present GRE data; however, some of them are not direct measurements of tissue susceptibility. The commonly used magnitude hypointensity ( $\mathrm{T} 2{ }^{*}$-weighted) and phase contrast at a given voxel depend on not only the tissue susceptibility in that voxel but also that of the nearby voxels in a convoluted manner, as well as imaging parameters, including field strength, TE, and object orientation. These blooming artifacts are problematic for depicting MS lesions ${ }^{27}$ but are addressed in QSM by deconvolving GRE phase data with the dipole kernel that connects tissue susceptibility with the magnetic field estimated from the GRE phase. ${ }^{22,23}$

In this study, we tried to connect QSM, a potential new biomarker for assessing inflammation in MS, with Gd enhancement, which has been established in the clinical literature as a surrogate indicator for inflammation. ${ }^{4}$ It seems that there is enough temporal correlation between the 2 aspects of inflammation activityBBB leakage and myelin debris removal/iron accumulation. This correlation may explain the very encouraging diagnostic sensitivity and specificity observed in this study when using only QSM to identify enhancing lesions in serial MR imaging examinations of new MS lesions. The evolution of an individual lesion in an MS brain may be regarded as independent from other lesions in the same MS brain, ${ }^{26}$ which may explain the observed similar areas under the curve for both jackknifing and bootstrapping receiver operating characteristic analysis.

This study has several limitations: 1) It was limited to assessing new enhancing lesions without Gd by using QSM in serial MR imaging. MS lesions older than 5 years may be chronically silent and QSM-isointense, ${ }^{19}$ confounding the interpretation of acute 
lesions that are also QSM isointense on the first or a single MR imaging. This outcome would limit the role of QSM to monitoring new lesions in serial or longitudinal MR imaging. This serious limitation requires us to continue seeking other non-contrast agent MR imaging features that differentiate old chronic lesions from new enhancing ones. Alternatively, because T1WI+Gd reflects the BBB leakage and QSM reflects myelin debris removal and iron accumulation, it may be useful to integrate T1WI+Gd and QSM information to form a comprehensive score to characterize acute MS lesion activity. 2) The sensitivity was not perfect because some new enhancing lesions demonstrated moderate hyperintensity on QSM, most of which $(82.3 \%, 14 / 17)$ were shellenhancing on T1WI+Gd instead of the common nodularenhancing type. Shell-enhancing lesions may be considered in the late stage of enhancing lesions, ${ }^{26,37,38}$ when myelin debris with negative susceptibility is being removed from the lesion and enters the peripheral circulation. ${ }^{16,17}$ 3) While QSM data are acquired by using the widely available $3 \mathrm{D}$ gradient-echo sequence and are processed in an automated manner, MS lesion susceptibility value measurement required manually drawing an ROI, which is laborious and may be alleviated by automated or semiautomated MS lesion ROI drawing tools. 4) This study is limited in sample size. Future studies should include applying the susceptibility cutoff value identified here to a larger cohort of patients with MS for evaluating the diagnostic accuracy in identifying new enhancing lesions.

\section{CONCLUSIONS}

QSM can be used in routine serial MR imaging monitoring of patients with MS to accurately identify the BBB leakage of new T2WI lesions without the use of a gadolinium contrast agent.

Disclosures: Susan A. Gauthier-UNRELATED: Consultancy: Biogen, Genentech, Genzyme; Grants/Grants Pending: Biogen,* Genzyme,, Mallincrodt,* Novartis,* EMD Serono.* Ajay Gupta—UNRELATED: Grants/Grants Pending: Foundation of the American Society of Neuroradiology Scholar Award, * AUR-Radiology Research Academic Fellowship award.* Weiwei Chen—RELATED: Grant: This study is partly supported by the National Natural Science Foundation of China (grant number 81401390).* Yi Wang-RELATED: Grant: National Institutes of Health (R01EB013443, R01NS090464)*; UNRELATED: Patents (planned, pending or issued): Cornell University, ${ }^{*}$ Comments: I am one of the inventors on the QSM patent. No money has been paid yet; Stock/Stock Options: Medimagemetric, Comments: partner ownership of Medimagemetric LLC, which is interested in QSM commercialization. No money paid. *Money paid to the institution.

\section{REFERENCES}

1. Trapp BD, Peterson J, Ransohoff RM, et al. Axonal transection in the lesions of multiple sclerosis. $N$ Engl J Med 1998;338:278-85 CrossRef Medline

2. McFarland HF, Martin R. Multiple sclerosis: a complicated picture of autoimmunity. Nat Immunol 2007;8:913-19 CrossRef Medline

3. Lublin FD, Reingold SC. Defining the clinical course of multiple sclerosis: results of an international survey: National Multiple Sclerosis Society (USA) Advisory Committee on Clinical Trials of New Agents in Multiple Sclerosis. Neurology 1996;46:907-11 CrossRef Medline

4. Polman CH, Reingold SC, Banwell B, et al. Diagnostic criteria for multiple sclerosis: 2010 revisions to the McDonald criteria. Ann Neurol 2011;69:292-302 CrossRef Medline

5. Vellinga MM, Oude Engberink RD, Seewann A, et al. Pluriformity of inflammation in multiple sclerosis shown by ultra-small iron oxide particle enhancement. Brain 2008;131:800-07 CrossRef Medline

6. Ladewig G, Jestaedt L, Misselwitz B, et al. Spatial diversity of blood- brain barrier alteration and macrophage invasion in experimental autoimmune encephalomyelitis: a comparative MRI study. Exp Neurol 2009;220:207-11 CrossRef Medline

7. Kanda T, Osawa M, Oba $\mathrm{H}$, et al. High signal intensity in dentate nucleus on unenhanced T1-weighted MR images: association with linear versus macrocyclic gadolinium chelate administration. $R a$ diology 2015;275:803-09 CrossRef Medline

8. Radbruch A, Weberling LD, Kieslich PJ, et al. Gadolinium retention in the dentate nucleus and globus pallidus is dependent on the class of contrast agent. Radiology 2015;275:783-91 CrossRef Medline

9. Roccatagliata L, Vuolo L, Bonzano L, et al. Multiple sclerosis: hyperintense dentate nucleus on unenhanced T1-weighted MR images is associated with the secondary progressive subtype. Radiology 2009; 251:503-10 CrossRef Medline

10. Shinohara RT, Goldsmith J, Mateen FJ, et al. Predicting breakdown of the blood-brain barrier in multiple sclerosis without contrast agents. AJNR Am J Neuroradiol 2012;33:1586-90 CrossRef Medline

11. Treabă CA, Bălaşa R, Podeanu DM, et al. Cerebral lesions of multiple sclerosis: is gadolinium always irreplaceable in assessing lesion activity? Diagn Interv Radiol 2014;20:178-84 CrossRef Medline

12. Blystad I, Håkansson I, Tisell A, et al. Quantitative MRI for analysis of active multiple sclerosis lesions without gadolinium-based contrast agent. AJNR Am J Neuroradiol 2016;37:94-100 CrossRef Medline

13. Lo CP, Kao HW, Chen SY, et al. Comparison of diffusion-weighted imaging and contrast-enhanced T1-weighted imaging on a single baseline MRI for demonstrating dissemination in time in multiple sclerosis. BMC Neurol 2014;14:100 CrossRef Medline

14. Lee SC, Moore GR, Golenwsky G, et al. Multiple sclerosis: a role for astroglia in active demyelination suggested by class II MHC expression and ultrastructural study. J Neuropathol Exp Neurol 1990;49: 122-36 CrossRef Medline

15. Brück W, Porada P, Poser S, et al. Monocyte/macrophage differentiation in early multiple sclerosis lesions. Ann Neurol 1995;38: 788-96 CrossRef Medline

16. Mehta V, Pei W, Yang G, et al. Iron is a sensitive biomarker for inflammation in multiple sclerosis lesions. PLoS One 2013;8:e57573 CrossRef Medline

17. Kutzelnigg A, Lassmann H. Pathology of multiple sclerosis and related inflammatory demyelinating diseases. Handb Clin Neurol 2014;122:15-58 CrossRef Medline

18. Cairo G, Recalcati S, Mantovani A, et al. Iron trafficking and metabolism in macrophages: contribution to the polarized phenotype. Trends Immunol 2011;32:241-47 CrossRef Medline

19. Chen W, Gauthier SA, Gupta A, et al. Quantitative susceptibility mapping of multiple sclerosis lesions at various ages. Radiology 2014;271:183-92 CrossRef Medline

20. Wiggermann V, Hernández Torres E, Vavasour IM, et al. Magnetic resonance frequency shifts during acute MS lesion formation. $\mathrm{Neu}$ rology 2013;81:211-18 CrossRef Medline

21. Zhang Y, Gauthier SA, Gupta A, et al. Longitudinal change in magnetic susceptibility of new enhanced multiple sclerosis (MS) lesions measured on serial quantitative susceptibility mapping (QSM). J Magn Reson Imaging 2016 Jan 22. [Epub ahead of print] CrossRef Medline

22. de Rochefort L, Liu T, Kressler B, et al. Quantitative susceptibility map reconstruction from $\mathrm{MR}$ phase data using Bayesian regularization: validation and application to brain imaging. Magn Reson Med 2010; 63:194-206 CrossRef Medline

23. Wang Y, Liu T. Quantitative susceptibility mapping (QSM): decoding MRI data for a tissue magnetic biomarker. Magn Reson Med 2015;73:82-101 CrossRef Medline

24. Liu J, Liu T, de Rochefort L, et al. Morphology enabled dipole inversion for quantitative susceptibility mapping using structural consistency between the magnitude image and the susceptibility map. Neuroimage 2012;59:2560-68 CrossRef Medline

25. Jenkinson $M$, Bannister $P$, Brady $M$, et al. Improved optimization for 
the robust and accurate linear registration and motion correction of brain images. Neuroimage 2002;17:825-41 CrossRef Medline

26. Gaitán MI, Shea CD, Evangelou IE, et al. Evolution of the bloodbrain barrier in newly forming multiple sclerosis lesions. Ann Neurol 2011;70:22-29 CrossRef Medline

27. Wisnieff C, Ramanan S, Olesik J, et al. Quantitative susceptibility mapping (QSM) of white matter multiple sclerosis lesions: interpreting positive susceptibility and the presence of iron. Magn Reson Med 2015;74:564-70 CrossRef Medline

28. Haacke EM, Makki M, Ge Y, et al. Characterizing iron deposition in multiple sclerosis lesions using susceptibility weighted imaging. J Magn Reson Imaging 2009;29:537-44 CrossRef Medline

29. Khalil M, Enzinger C, Langkammer C, et al. Quantitative assessment of brain iron by $\mathrm{R}(2)^{\star}$ relaxometry in patients with clinically isolated syndrome and relapsing-remitting multiple sclerosis. Mult Scler 2009;15:1048-54 CrossRef Medline

30. Ropele S, de Graaf W, Khalil M, et al. MRI assessment of iron deposition in multiple sclerosis. J Magn Reson Imaging 2011;34:13-21 CrossRef Medline

31. Khalil M, Langkammer C, Ropele S, et al. Determinants of brain iron in multiple sclerosis: a quantitative 3T MRI study. Neurology 2011; 77:1691-97 CrossRef Medline
32. Hagemeier J, Heininen-Brown M, Poloni GU, et al. Iron deposition in multiple sclerosis lesions measured by susceptibility-weighted imaging filtered phase: a case control study. J Magn Reson Imaging 2012;36:73-83 CrossRef Medline

33. Paling $\mathrm{D}$, Tozer $\mathrm{D}$, Wheeler-Kingshott $\mathrm{C}$, et al. Reduced $\mathrm{R} 2$ ' in multiple sclerosis normal appearing white matter and lesions may reflect decreased myelin and iron content. J Neurol Neurosurg Psychiatry 2012;83:785-92 CrossRef Medline

34. Bagnato F, Hametner S, Welch EB. Visualizing iron in multiple sclerosis. Magn Reson Imaging 2013;31:376-84 CrossRef Medline

35. Bian W, Harter $\mathrm{K}$, Hammond-Rosenbluth $\mathrm{KE}$, et al. A serial in vivo 7T magnetic resonance phase imaging study of white matter lesions in multiple sclerosis. Mult Scler 2013;19:69-75 CrossRef Medline

36. Absinta M, Sati P, Gaitan MI, et al. Seven-Tesla phase imaging of acute multiple sclerosis lesions: a new window into the inflammatory process. Ann Neurol 2013;74:669-78 CrossRef Medline

37. Cotton F, Weiner HL, Jolesz FA, et al. MRI contrast uptake in new lesions in relapsing-remitting MS followed at weekly intervals. Neurology 2003;60:640 - 46 CrossRef Medline

38. Guttmann CR, Ahn SS, Hsu L, et al. The evolution of multiple sclerosis lesions on serial MR. AJNR Am J Neuroradiol 1995;16:1481-91 Medline 